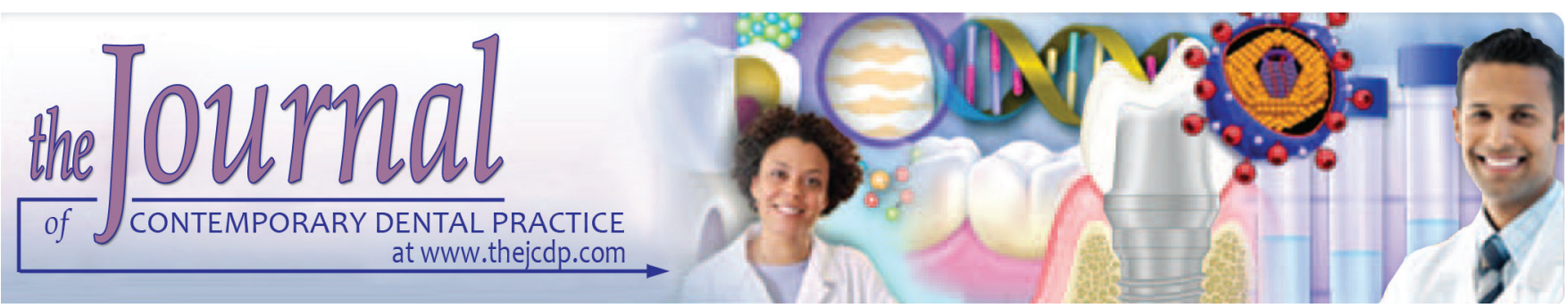

\title{
A Novel Mobile Health Approach to Early Diagnosis of Oral Cancer
}

\author{
${ }^{1} \mathrm{~N}$ Praveen Birur, ${ }^{2}$ Sanjana Patrick, ${ }^{3}$ Suchitra Bajaj, ${ }^{4}$ Shubhasini Raghavan, ${ }^{5}$ Amritha Suresh, ${ }^{6}$ Sumsum P Sunny \\ ${ }^{7}$ Radhika Chigurupati, ${ }^{8}$ Petra Wilder-Smith, ${ }^{9}$ Keerthi Gurushanth, ${ }^{10}$ Shubha Gurudath, ${ }^{11}$ Pratima Rao \\ ${ }^{12}$ Moni A Kuriakose
}

\section{ABSTRACT}

Aim: The incidence of oral cancer is high in India, which can be reduced by early detection. We aimed to empower frontline health care providers (FHP) for early detection and connect specialist to rural population through mHealth.

Materials and methods: We provided training to FHPs in examination of oral cavity, use of mobile phone for image capture, and risk factor analysis. The FHPs were selected from different cohorts in resource-constrained settings. The workflow involved screening of high-risk individuals in door-to-door and workplace settings, and capture of images of suspected lesions. Uploaded data were interpreted and recommendation was sent by specialist from a remote location. Their recommendation was

${ }^{1}$ Department of Oral Medicine and Radiology, KLE Society's Institute of Dental Sciences, Bengaluru, Karnataka, India; Oral Cancer Screening, Biocon Foundation, Bengaluru, Karnataka India

${ }^{2}$ Department of Oral Cancer Screening, Biocon Foundation Bengaluru, Karnataka, India

${ }^{3,11}$ Department of Health Care, Biocon Foundation, Bengaluru Karnataka, India

4,9,10 Department of Oral Medicine and Radiology, KLE Society's Institute of Dental Sciences, Bengaluru, Karnataka, India

${ }^{5,6}$ Department of Head and Neck Oncology, Mazumdar Shaw Center for Translational Research, Mazumdar Shaw Medical Center, Bengaluru, Karnataka, India

${ }^{7}$ Department of Oral and Maxillofacial Surgery, Boston University Medical Campus, Boston, Massachusetts, USA

${ }^{8}$ Department of Dentistry, Beckman Laser Institute, University of California, Irvine, California, USA

${ }^{12}$ Department of Head and Neck Oncology, Cochin Cancer Research Center, Ernakulum, Kerala, India

Corresponding Author: N Praveen Birur, Department of Oral Medicine and Radiology, KLE Society's Institute of Dental Sciences, Bengaluru, Karnataka, India; Oral Cancer Screening Biocon Foundation, Bengaluru, Karnataka, India, Phone: +919845136960, e-mail: praveen.birur@biocon.com intimated to FHPs who arranged for further action. Two more initiatives, one for multiple dental schools and another for private practitioners, were undertaken.

Results: During the period from 2010 to $2018,42,754$ subjects have been screened, and 5,406 subjects with potentially malignant disorders have been identified. The prevalence of potentially malignant disorders varied from 0.8 to $62 \%$ at different cohorts; 516 biopsies have been performed at remote locations.

Conclusion: Connecting specialists to rural population was made possible through the use of mobile health. Trained FHP were able to reach out to the population. Electronic data capture facilitated efficient follow-up. The program was very cost-effective with screening completed under $\$ 1$ per person.

Clinical significance: In view of the high incidence of oral cancer in India, and the resource-constrained settings, mobile health paves the way for better access to specialist care for the rural population.

Keywords: Frontline health care providers, Mobile health, Oral cancer, Oral potentially malignant disorders, Remote diagnosis.

How to cite this article: Birur NP, Patrick S, Bajaj S, Raghavan S, Suresh A, Sunny SP, Chigurupati R, Wilder-Smith $P$, Gurushanth K, Gurudath S, Rao P, Kuriakose MA. A Novel Mobile Health Approach to Early Diagnosis of Oral Cancer. J Contemp Dent Pract 2018;19(9):1122-1128.

Source of support: Nil

Conflict of interest: None

\section{INTRODUCTION}

The burden of noncommunicable diseases is increasing in India. One among these is cancer, the cause of $15 \%$ of all deaths. ${ }^{1}$ Worldwide, oral cancer is the 11 th most common cancer among men, and 15th among women. ${ }^{2}$ However, it is ranked first among men and third among women in India, ${ }^{3}$ where high incidence rates of 20 per 100,000 population have been reported. ${ }^{4}$ 
The public health care system in India consists of a three-tier system composed of subcenters, primary health centers (PHCs), and community health centers. However, infrastructure and staffing are inadequate in these public health care centers. ${ }^{5}$ Public sector tertiary care institutions provide specialized services, and also serve as primary care providers to compensate for weaknesses in the public health sector. ${ }^{6}$ However, they function independently of the primary health care system.

The private health care sector is extensive, heterogeneous, and largely unregulated, including sophisticated hospitals with advanced investigatory and treatment facilities. However, these may not be within the reach of lower economic sectors of the population. The distribution of skilled manpower is exceedingly unequal in India, with only $3.3 \%$ of all allopathic doctors working in public health facilities in rural areas. Informal care providers, with no formal medical training or government registration for medical practice are estimated to represent 55\% of all providers. ${ }^{7}$ They frequently act as the first point of contact for health-related issues, especially in rural areas. ${ }^{6}$ Practitioners using indigenous systems of healing are also prevalent outside of urban centers.

Prognosis and treatment outcomes in terms of morbidity and mortality are excellent if oral cancer is detected before metastasis, with $82.8 \%$ surviving 5 years after diagnosis. However, most oral cancers are detected after metastasis, when 5-year survival is only $27.8 \%{ }^{8}$ Because oral cancer and oral potentially malignant disorders (OPMDs) are asymptomatic, the majority of patients do not present for care until a late stage has been reached. In spite of awareness about tobacco use and its side effects, patients are unsure of the symptoms that warrant an approach to a doctor and try out home remedies in favor of visit to doctors.

Clearly, it is not effective to wait for patients to report to doctors at primary levels. It is essential for doctors to reach out to patients, even in underserved areas. However, in view of the large population, low doctor-patient ratio, and geographic difficulties, it may not be possible for specialists to visit every patient. A more realistic concept is that of empowering health workers. Existing resources in the form of FHPs, such as Accredited Social Health Activists (ASHA) can be utilized for screening, and to provide linkage to specialist care. This program was planned to determine the proportion of subjects with OPMDs and at risk for development of oral cancer across various geographic locations utilizing FHPs.

Through the current mHealth program, we hope to achieve decrease in prevalence of OPMDs and downstage oral cancer by creating awareness, early detection, treatment, and timely referral.

\section{METHODS AND RESULTS}

Based on this concept of empowering FHPs through mobile health (mHealth), we conducted a pilot study in a targeted cohort screened by FHPs and an opportunistic cohort screened by dental professionals. ${ }^{9}$ The work flow involved the use of mobile phone with oral cancer screening software, Sana. All uploaded data, text and images, were viewed in Open Medical Record System (Open MRS), an open-source web interface. The specialist could interpret the images on a monitor and had the provision to zoom in, adjust contrast or request for a retake. The outcomes measured were lesion detection and capture of interpretable images of oral lesions. A remote specialist, essentially an oral medicine specialist, looked at the risk factors for oral cancer and classified OPMDs based on site, size, shape, and color. Recommendations of either suspicious or nonsuspicious lesions were given; $61 \%$ of images obtained by FHPs were deemed interpretable; and $45 \%$ of these were confirmed to be OPMDs. In the opportunistic cohort, all images captured were interpretable and were confirmed as OPMDs. ${ }^{9}$

This pilot study demonstrated that mHealth-based approach can aid remote early detection of oral cancer. It mapped out a novel approach that can provide the basis for a paradigm shift in cancer care and outcomes in low-resource settings, through networking FHPs with local community cancer screening service. The following conclusions were drawn (Flow Chart 1):

- At the community level, trained FHPs are capable of utilizing smart phone-based technology for screening the oral cavity. It was suggested that further training of FHP with newer technology needs to be conducted.

- Involvement of a dental school as a nodal center acted as a triage point for further specialist referral. In this pilot study, the dental school with a geographic

Flow Chart 1: Plan of action drawn following pilot study

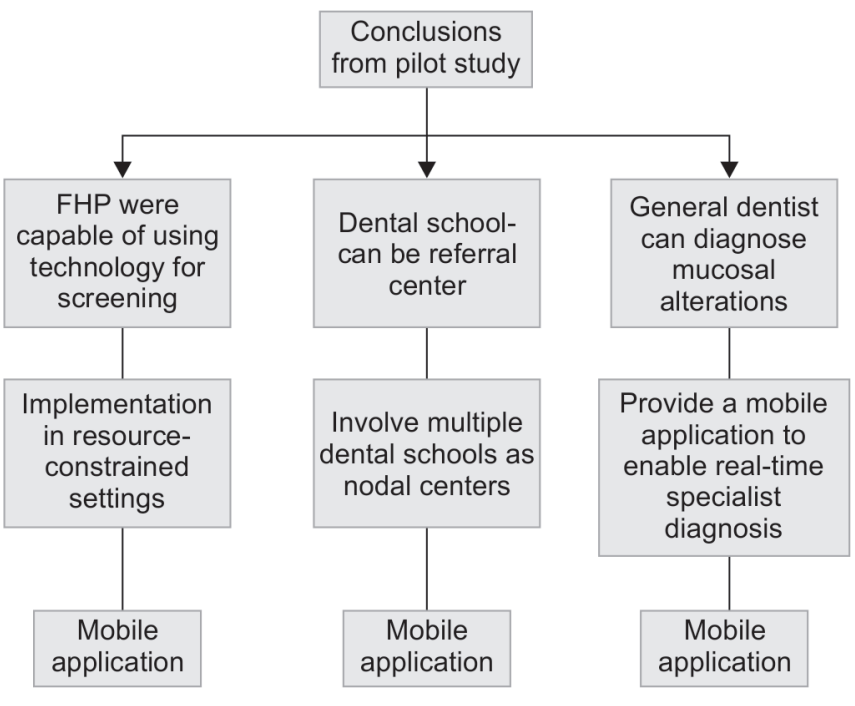


proximity to the study cohort was involved. The dental school acts as an intermediary, manages patients with OPMD rather than referring to tertiary center. This was also effective in creating awareness in the screened population at minimal cost. In view of the large number of dental schools spread across India, involving other dental schools would be an excellent option.

- Based on this study, a mobile phone application for general dentists was designed and launched to assist them in managing oral cancer in diverse patient populations.

As the next action plan, the following screening programs were initiated after obtaining ethics clearance from the Institution Ethics Committee, KLE Society's Institute of Dental Sciences, Bengaluru, India.

\section{Implementation in Resource-constrained Settings}

As part of its community and social responsibility engagement, Biocon Foundation has spearheaded the campaign against oral cancer through screening of rural populations using mHealth. For this project, a bifurcated screening approach is being used: Door-to-door and workplace. To date, 42,754 subjects have been individually approached with the aid of technology. Of these, 20,462 subjects have been screened by door-to-door screening, and 2,650 subjects have been diagnosed with OPMDs. In various workplace settings, 22,292 subjects have been screened, and 2,756 subjects diagnosed with OPMDs (Flow Chart 2 and Graph 1). The prevalence of OPMDs ranged from 0.8 to $62 \%$ at different cohorts, with a mean of $12.6 \% ; 67$ and 449 biopsies have been performed in door-to-door and workplace settings respectively, underlining the challenges of performing invasive procedures in these settings.

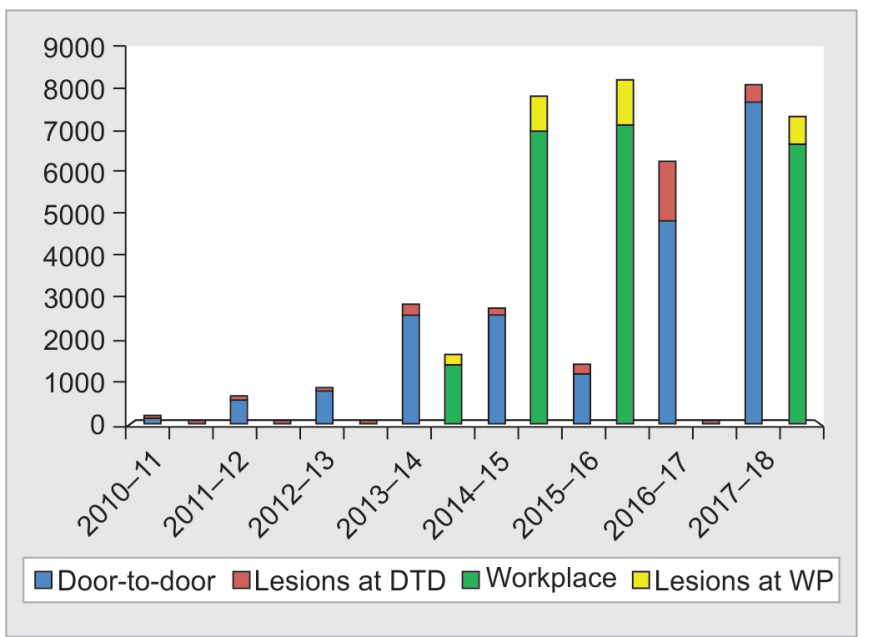

Graph 1: Annual progress of mHealth program
Flow Chart 2: mHealth screening program outline

\begin{tabular}{|c|c|}
\hline 2010-11 & $\begin{array}{l}\text { - Pilot study, door-to-door screening } \\
\text { - Screening in two villages } \\
\text { - } 130 \text { persons screened; } 23 \text { lesions detected }\end{array}$ \\
\hline 2011-12 & $\begin{array}{l}\text { - Door-to-door screening by biocon employees } \\
\text { - Uploads from biocon clinics } \\
\text { - } 583 \text { persons screened; } 67 \text { lesions detected }\end{array}$ \\
\hline $2012-13$ & $\begin{array}{l}\text { - Door-to-door screening } \\
\text { - } 781 \text { persons screened; } 41 \text { lesions detected }\end{array}$ \\
\hline 2013-14 & $\begin{array}{l}\text { - Door-to-door and workplace screening } \\
\text { - } 2609 \text { persons screened; } 253 \text { lesions detected in door- } \\
\text { to-door and } 1436 \text { persons screened; and } 191 \text { lesions } \\
\text { detected in workplace }\end{array}$ \\
\hline 2014-15 & $\begin{array}{l}\text { - Workplace screening } \\
\text { - } 7002 \text { persons screened; } 822 \text { lesions detected } \\
\text { - Door-to-door screening } \\
\text { - } 2623 \text { persons screened; } 167 \text { lesions detected } \\
\text { - Follow-up screening in } 2 \text { villages, } 21 \text { new cases } \\
\text { detected }\end{array}$ \\
\hline
\end{tabular}

-Workplace screening

- Students from community trained and employed for

2015-16. Screening

- Door-to-door screening

- 1177 persons screened; 235 lesions detected

- Screen and treat program initiated

2016-17 - Screening in Telgi, Yediyur, and Sawai Madhopur

- 4845 persons screened; 1466 lesions detected

- Door-to-door screening

- 7714 persons screened; 398 lesions detected

- Workplace screening

- 6695 persons screened; 664 lesions detected

The Biocon mHealth program was first implemented in 2011. We developed a standard 2-day training module for oral cancer screening and use of mobile phone application, through PowerPoint, videos, and clinical cases. Training was given by oral medicine specialists, and FHPs knowledge was tested at the end. In 2011, door-to-door screening of high-risk persons was performed in two villages by four Biocon employees trained as FHPs. Mobile phones equipped with cameras for documentation as well as the decision-supported algorithm, Poi Mapper, were issued to these FHPs (Flow Chart 3). While the screening program was generally effective in identifying at-risk patients and capturing quality intraoral images, uploads were challenging in many locations due to poor connectivity, so that uploads were typically made from Biocon clinics in the villages.

During the period from 2013 to 2014, three additional villages and four workplace settings were added to the program. FHPs were proficient at identification of lesions and image captures. During the period from 2014 to 2015, a comparison of smokeless tobacco habits and oral lesions was performed at a workplace setting in conjunction with 


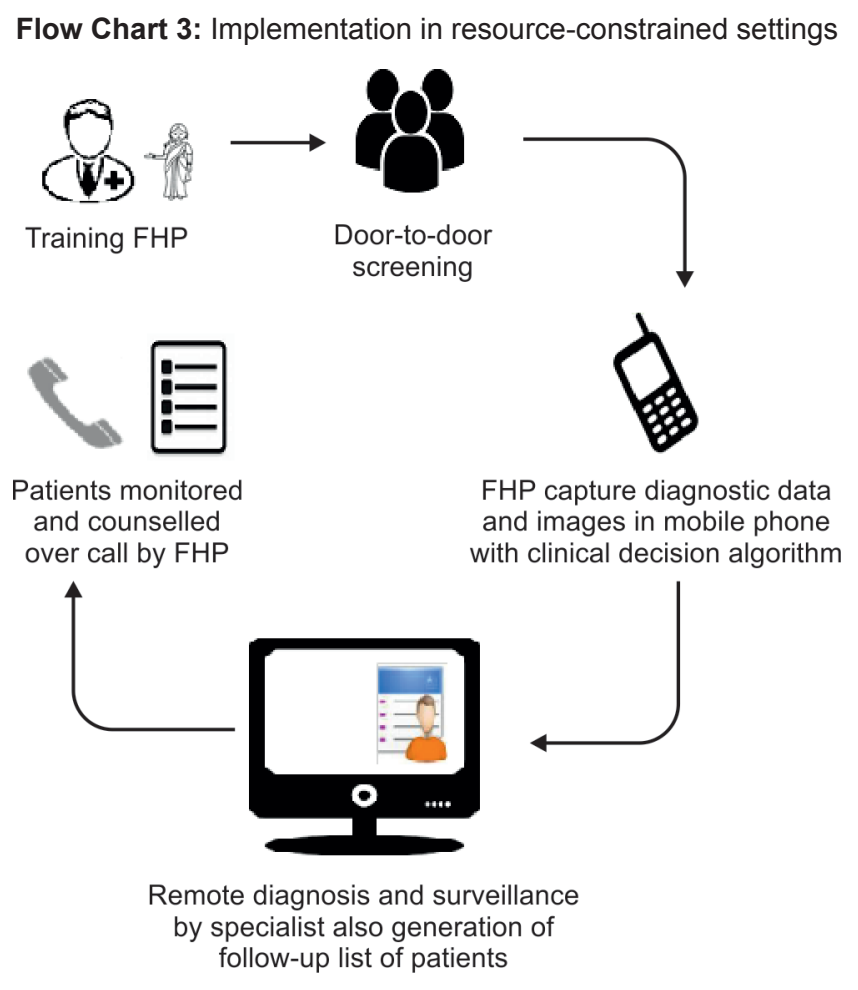

salivary analysis. ${ }^{10,11} \mathrm{~A}$ follow-up screening was carried out in two villages 2 years after the first visit; 21 new lesions were detected; however, there were no advanced lesions. During the period from 2015 to 2016, a scaledup model was implemented, and our reach extended to North-East India. Here, students from the local community were trained and employed for screening. However, their involvement was not effective with regard to screening accuracy and image quality. Wired data transfer was attempted as a temporary measure, due to very poor connectivity in these areas. In our full-fledged program in North-East India, which involved population-based and screening at schools, 7,202 subjects were covered, 311 OPMDs were identified, and subjects were recalled for biopsy. The program is currently underway; 30 biopsies were performed in which one subject each with squamous cell carcinoma and microinvasive carcinoma were detected. These two subjects were advised to undergo wide excision, as they had $\mathrm{T} 1$ lesions.

In India, it is critical to integrate any oral cancer control program with government-run health initiatives. The ambitious "screen and treat" program was initiated by Biocon Foundation in association with the Government of Karnataka. It is designed to reach out to rural populations through existing health care resources and ASHAs. The ASHAs were trained in the standard format. They underwent an objective pre- and posttraining test during training program and knowledge retention was assessed after 3 months.

The "screen and treat" program was conducted in two phases: In the first, persons served by a PHC aged 18+

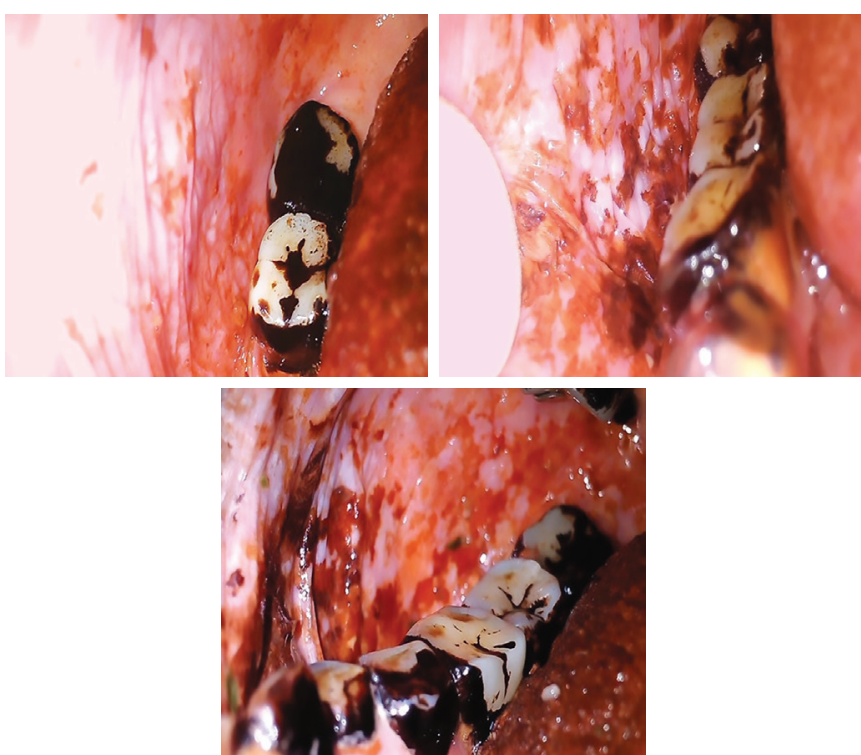

Fig. 1: User interface, Open MRS, for specialist recommendation

underwent thorough door-to-door screening to identify at-risk individuals. Using electronic data capture with digital sheets, data were automatically integrated into a database and tabulated. All subjects with high-risk habits as well as those with a positive family history of cancer were recalled to the PHC for further evaluation. In the second phase at the PHC, patients received a general health screening using a Multi-ParameterPatient-Monitor device to assess their general health status. A local coordinator at the PHC examined the patients, identified oral lesions, and uploaded findings using the Poi Mapper application. The uploaded images were interpreted by an oral medicine specialist (Fig. 1), and results or requests for further investigations and treatment options were communicated to the FHP. They in turn would carry out the necessary actions. This program was conducted at two villages in Karnataka and at noncommunicable diseases desks at Rajasthan. In total, 4845 subjects have been screened and 1,466 lesions have been identified.

\section{Oncogrid}

Another parallel program called "Oncogrid" had the goal of assessing the routine "standard of care" during screening carried out in dental schools compared with mHealth-based screening. Screening was planned to be carried out in seven different dental schools across the state of Karnataka. The schools acted as nodal centers, with surrounding villages targeted for screening. Students pursuing undergraduate programs and members of the Departments of Oral Medicine and Public Health were actively involved in screening. Four dental schools actively participated. A total of 1,444 subjects were screened. Intraoral images were uploaded using 
the mobile application Emocha. In addition to collecting demographics, this app required the upload of ten images from each subject, covering the entire oral cavity irrespective of the presence of lesions; 234 smokers and 564 smokeless tobacco users were identified; 93 abnormal findings were identified by FHP.

\section{Empowerment of General Dentists}

It was deemed necessary to reach out to general dental practitioners, and empower clinicians in diagnosing asymptomatic oral mucosal alterations. Several studies indicate that dentists lack knowledge in the area of oral cancer etiology and diagnosis. ${ }^{12}$ An application termed "Experteez" was downloaded onto the mobile phones of the dentists participating in this study. The application has a workflow of questions regarding adverse habits, with provision to record photographs of lesions. The dentists obtained this information and uploaded the data. Selected experts in the field of oral medicine with the same application on their phones received the data and provided their opinion regarding diagnosis, treatment options, and referral. Thus, there was direct communication between two mobile phone users, rather than at the back-end. This real-time consultation saves chair-side time, provides immediate input about mucosal lesions and their management, and negates the need for further appointments. The application was launched at the International Conference of the Federation of Head and Neck Oncology in 2016. In the pilot study, 43 subjects were examined, and there was substantial agreement between experts. However, use of the application by private dentists has not met the expectation, with low usage and low number of uploads.

\section{DISCUSSION}

Oral cancer and precancers are ideally suited to screening and early diagnosis as they are accessible to direct examination, and are associated with clearly identifiable risk factors. ${ }^{13}$ In spite of this, oral cancer is common in India. This is attributed to the high prevalence (about $35 \%$ of adults) of tobacco use in the country. ${ }^{14}$ There is a great opportunity to bring down prevalence rates through community screening, early detection, and timely referral.

In recent years, there has been a tremendous growth in mobile phone usage in developing countries. Mobile phone subscribers in developing countries outnumber those in developed countries. ${ }^{15}$ There are several examples for the successful implementation of mobile health programs in various scenarios. ${ }^{16,17}$ The authors have also conducted mHealth programs. ${ }^{9,10}$ It was considered opportune to share the experience and highlight the challenges faced in the actual implementation of these programs.

\section{Implementation Challenges}

One of the key factors in successfully implementing such programs at a grassroots level is to secure the active buy-in and involvement of local authorities who, in our experience, were predominantly keen to participate. The FHPs selected were residents in the community where the screenings were conducted. The ASHAs became involved through government agencies and were generally effective in covering their respective populations.

A standard training module was developed to train the FHPs for measuring outcomes related to fieldwork performance. Posttraining dropout of FHPs after program initiation caused unexpected delays in running the program. It was a challenge to identify new FHPs and train them in a timely fashion.

In the field, most subjects were cooperative, especially in rural populations. The participants were counseled on maintaining oral health and a toothpaste and antioxidants were given when required. Reasons for not participating varied from the absence of male members of the family, social stigmas, "lack of time" in workplace settings to their "perception of being healthy." Some presumptions included "absence of lesions even after years of chewing," "tobacco gives relief from toothache," and "it's a daily routine." Many patients with lesions stated that they had used "more lime and that the lesion would regress by itself." Screening within houses added the challenges of lack of clinic-like environment and poor lighting. Male breadwinners were generally absent during door-todoor screening, and were recruited during evenings and weekends. It was noted that a recommendation to get themselves screened, by an authority they trusted, such as a PHC doctor, gave better results.

Any subject with an identifiable OPMD was recalled to a PHC or the nearest facility for a biopsy. However, there was poor compliance to the recall. We assessed the reasons for the same in one cohort. It was found that $459 / 1,871$ subjects were unwilling to travel the distance for a biopsy. Subjects did not consent to an invasive procedure, were fearful of injections, blood, and the resulting wound.

\section{Technical Challenges}

The success of this mHealth initiative to provide widespread screening of underserved population relied on technical aspects to a great extent.

Traditionally, field-based data collection or surveys use paper and pen-based methods in developing countries. Physical transportation of completed forms as well as consolidation, transfer, management, and analysis of the data involve considerably greater time and cost than for electronically collected data. ${ }^{16}$ These limitations encouraged the development of electronic data collection 
approaches. Our mHealth initiatives were conducted to evaluate the feasibility and effectiveness of mobile application-based diagnosis. It ensured screening of thousands of persons in rapid turnaround time. It was also successful in terms of logistics, electronic data capture, and empowerment of local resources.

All uploaded subject data were deidentified and stored to a secure server at Biocon Foundation. It was desirable to keep the size of the uploads to a minimum and photos were limited to capture only the positive sites. The quality of images captured varied based on the distance from which the photograph was taken, zooming of the screen, etc. The quality of photos obtained by FHP improved with training and were generally acceptable. The images obtained at workplace settings were better than those obtained from door-to-door screening.

Subjects were identified using multiple identifiers, such as demographic details, phone number, and ID cards. In spite of these, some difficulty was encountered when subjects were not aware of their date of birth, or phone number, or when they lost their ID cards. In most cases, this was resolved through matching other identifiers.

The oral medicine specialist had to commit time for remote diagnosis. Complete subject data were not available at times. The remote specialist's recommendation was transmitted to the FHP, who then recruited the subject for further steps. However, this transformation took time due to migration of subjects, or absence of contact numbers.

\section{Administrative Challenges}

It is advisable to prioritize geographic location of screening based on disease burden, local needs, and consideration for social and cultural barriers. The budget outlay for preventive oral cancer program should be designed such that screening is completed within $\$ 1$ per person. This is crucial to make the program cost-effective, so that it can be scaled up. On an average, Biocon Foundation spends about Rs. 59 per person inclusive of screening, chemoprevention, creating awareness, and providing patient education.

The mean proportion of subjects with OPMDs was $12.6 \%$ in the screened population in our program. This is similar to the prevalence found in other studies. ${ }^{17-20}$ Detecting an OPMD early should be followed by monitoring and providing timely referral. Implementation of programs for monitoring of high-risk population is challenging and depends on the skill of the FHP. Recognizing the importance for follow-up, a surveillance module with biometric capture is being implemented.
Primary care physicians, ASHAs, dental schools, general dental practitioners, and tertiary care centers are all links in the chain of patient management. Hence, they need to be integrated and provided proper communication channels to monitor high-risk groups. Biocon Foundation, a voluntary organization, is making an effort in this direction by creating awareness among the populace regarding the ill-effects of a traditionally accepted habit, providing guidance for self-mouth examination, an individualized approach and providing timely referral.

The shortcomings noted in our series of programs include variable participation of dental schools in the Oncogrid trial, and poor response to the use of Experteez app for general dentists. Both these case scenarios emphasize the need for a core administrative center to implement technology in health care. Thus, our future recommendation is to provide "linkage to care" wherein rural population, nodal center, and tertiary center are interconnected to communicate. This will effectively reduce the burden at the tertiary center.

In this system, each individual has a predefined role to play in reducing the cancer burden. The FHP provides early detection, a general dental practitioner treats and provides referral, the dental school is responsible for performing a biopsy and surgical management, and the tertiary center provides advanced treatment and refers the patient back to the dental school for monitoring. This completes a linkage to care which ensures that a patient with an OPMD or Oral Cancer obtains optimal treatment and is not lost in the complex pathway of care.

\section{CONCLUSION}

This oral cancer screening program was conducted with a view to reach out to sections of the population which do not have access to specialist care. With the use of mobile applications and FHP, we have examined almost 50,000 people from across the country. Electronic data capture aided remote diagnosis and also maintenance of patient records for follow-up. Thus, use of technology can provide better access to health care in resourceconstrained settings.

\section{REFERENCES}

1. IHME. The global burden of cancer 2013. Seattle (WA): IHME; 2013. [cited 2016 Aug 13]. Available from: www.healthdata. org/research-article/global-burden-cancer-2013.

2. IARC. GLOBOCAN 2012: estimated cancer incidence, mortality and prevalence worldwide in 2012. Lyon: IARC; 2012. [cited 2016 Aug 13]. Available from: http:/ / globocan.iarc.fr.

3. Vigneswaran N, Williams MD. Epidemiological Trends in head and neck cancer and aids in diagnosis. Oral Maxillofac Surg Clin North Am 2014 May;26(2):123-141. 
4. Coelho KR. Challenges of oral cancer burden in India. J Cancer Epidemiol 2012 Oct;2012:701932.

5. Bhandari, L.; Dutta, S. Health Infrastructure in rural India. In: Kalra P, Rastogi A, editors. India infrastructure report 2007. New Delhi: Oxford University Press; 2007. p. 265-285.

6. Patel V, Parikh R, Nandraj S, Balasubramaniam P, Narayan K, Paul VK, Shiva Kumar AK, Chatterjee M, Srinath Reddy K. Assuring health coverage for all in India. Lancet 2015 Dec;386(10011):2422-2435.

7. Sudhinaraset M, Ingram M, Lofthouse HK, Montagu D. What is the role of informal healthcare providers in developing countries? A systematic review. PloS One 2013 Feb;8(2):e54978.

8. NIDCR. Oral cancer 5-year survival rates by race, gender, and stage of diagnosis. Surveillance, Epidemiology, and End Results (SEER) Program, National Cancer Institute Surveillance Research Program, based on November 2006 submission of SEER Series 9 (1996-2003). 2006. [cited 2017 Jul 29]. Available from: https://www.nidcr. nih.gov / DataStatistics / FindDataByTopic/OralCancer/ OralCancer5YearSurvivalRates.htm.

9. Birur PN, Sunny SP, Jena S, Kandasarma U, Raghavan S, Ramaswamy B, Shanmugam SP, Patrick S, Kuriakose R, Mallaiah J, et al. Mobile health application for remote oral cancer surveillance. J Am Dent Assoc 2015 Dec;146(12): 886-894.

10. Desai R, Birur P, Bajaj S, Shubhasini AR, Bhanushree R, Shubha G, Keerthi G, Tiwari V, Patrick S, Hegde U, et al. Smokeless tobacco-associated lesions: a mobile health approach. J Contemp Dent Prac 2015 Oct;16(10):813-818.

11. Hannien PE, Silvonen JJ, Martikkala E, Desai D, Teimonen T, Tiittanen SA, Mundill P, Mukundan C, Desai R, Birur P. A low resource setting oral lesion screening tool. Matters 2016 Mar:1-7.
12. Messadi DV, Wilder-Smith P, Wolinsky L. Improving oral cancer survival: the role of dental providers. J Calif Dent Assoc 2009 Nov;37(11):789-798.

13. Mashberg A, Samit A. Early diagnosis of asymptomatic oral and oropharyngeal squamous cancers. CA Cancer J Clin 1995 Nov-Dec;45(6):328-351.

14. Sridharan G. Epidemiology, control and prevention of tobacco induced oral mucosal lesions in India. Indian J Cancer 2014 Jan-Mar;51(1):80-85.

15. Ganesan M,PrashantS, Jhunjhunwala A. A review on challenges in implementing mobile phone based data collection in developing countries. J Health Inform Dev Ctries 2012;6(1):366-374.

16. Lim S, Xue L, Yen CC, Chang L, Chan HC, Tai BC, Duh HB, Choolani M. A study on Singaporean women's acceptance of using mobile phones to seek health information. Int J Med Inform 2011 Dec;80(12):e189-e202.

17. Surka S, Edirippulige S, Steyn K, Gaziano T, Puoane T, Levitt N. Evaluating the use of mobile phone technology to enhance cardiovascular disease screening by community health workers. Int J Med Inform 2014 Sep;83(9):648-654.

18. Kumar S, Debnath N, Ismail MB, Kumar A, Kumar A, Badiyani BK, Dubey PK, Sukhtankar LV. Prevalence and risk factors for oral potentially malignant disorders in Indian population. Adv Prev Med 2015 Jul;2015:208519.

19. Kumar YS, Acharya S, Pentapati KC. Prevalence of oral potentially malignant disorders on workers of Udupi taluk. South Asian J Cancer 2015 Jul-Sep;4(3):130-133.

20. Chher T, Hak S, Kallarakkal TG, Durward C, Ramanathan A, Ghani WM, Razak IA, Harun MH, Ashar NA, Rajandram RK, et al. Prevalence of oral cancer, oral potentially malignant disorders and other oral mucosal lesions in Cambodia. Ethn Health 2018 Jan;23(1):1-15. 